\title{
AUTONOMOUS INTELLIGENT AERIAL SEARCH AND RESCUE (ASAR) ROBOTS IN RESCUING MISSIONS
}

\author{
Suzana Stojaković - Čelustka ${ }^{385}$ \\ Reza Raeisis ${ }^{386}$ \\ Mohammad Nourbakhsh ${ }^{387}$
}

https://doi.org/10.31410/itema.2018.1123

\begin{abstract}
Rapid advances in technology are revolutionizing the roles of aerial, terrestrial and maritime robotic systems in disaster relief. Robots can be deployed quickly in areas deemed too unsafe for humans and are used to guide rescuers, collect data, deliver essential supplies or provide communication services.

Information plays a key role in crisis management and relief efforts for natural disaster and firefighting scenarios. Given their flight properties, UAVS (Unmanned Aerial Vehicles) provide new and interesting perspectives on the data gathering and processing for these purposes. A new generation of UAVs may help in rescuing missions in the way to be used to detect and enter damaged buildings, assisting rescue robots and responders on the ground by speeding up the search for survivors through prioritizing which areas to search first.

UAVs used for rescuing missions are most commonly powered by rechargeable batteries and are operated autonomously through onboard computers or by remote control. Their equipment typically comprises radar and laser scanners, multiple sensors and video and optical cameras as well as infrared cameras that are used to identify heat signatures of human bodies and other objects. This helps rescuers to locate survivors at night and in large, open environments and to identify hot spots from fires. Listening devices can pick up hard-to-hear audio, while Wi-Fi antennas and other attachments detect signals given off by mobile phones and plot a map that outlines the locations of victims.

Developing autonomous intelligent aerial search and rescue (ASAR) robots is faced with two main challenges. The first one is to achieve a sufficient autonomy level, both in terms of navigation and interpretation of the data sensed. The second major challenge relates to the reliability of the robot, with respect to accidental (safety) or malicious (security) risks. In the field of aerial rescuing robotics, big research challenges include also the design of a) robust enough hardware than can resist collisions and is easy to transport, b) new control methods for autonomous flight, $c$ ) innovative sensors for safe navigation and rescuing purposes, and d) fast interpretation and analysis of vital data collected through sensors.

This paper presents some of solutions to above mentioned challenges in design of ASAR robots for rescuing missions.
\end{abstract}

Keywords: disaster management, firefighting, UAV, robot, ASAR, autonomous, rescuing mission

\footnotetext{
${ }^{385}$ InfoSet d.o.o., Zagreb School of Economics and Management (ZSEM), Zagreb, Croatia

${ }^{386}$ InfoSet d.o.o, Tehran University of Medical Sciences (TUMS), Tehran, Iran

${ }^{387}$ InfoSet d.o.o., Islamic Azad University, Isfahan, Iran
} 


\section{INTRODUCTION}

Rescuing operations are often carried out in hazardous environments, depending on the characteristics, severity, and the extent of the disastrous event. Accurate estimates of the equipment to be sent to the affected areas, as well as the number of items and the requirements for rescuing activities are usually determined only upon the arrival of first rescuing forces to the field of accident. Given the traffic volumes of today, especially in the big cities, the deployment of rescuing forces to crisis-hit areas is often time-consuming. On the other hand, the short time of response in rescuing operations is of crucial importance.

The main purpose of using Aerial Search and Rescue robots (abbreviation ASAR will be used in further text) in rescuing operations is the provision of relevant data to the collecting system for the rapid acquisition and transmission of images from crisis areas to the command centers of rescuing teams.

The ASARs are able to fly at the shortest time to the accident area from the moment of receiving first alert message, enabling in this way fast response of ground rescuing crews. They are capable of flying to the most distant endangered areas at very short time periods, e.g. in 2 to 3 minutes from first alarm. At the same time, land vehicles might need at least ten minutes to travel across the same distance. As soon as the ASAR arrives in the area with its installed camera, having the infrastructure for sending images online, the images of the affected area are transmitted to the command centers and are helping the rescuing crews to make fast and accurate decisions about rescuing activities.

On the other hand, the ASAR can fly on the route to endangered areas to help rescuing vehicles by sending the images of the route to the cars and the command center. In that way rescuing cars can at any moment determine their optimal routes and prevent traffic jamming and other traffic problems.

The ASAR can also carry rescuing packages to the affected areas. This feature, especially in the cases of severe fires or earthquakes with possibly blocked rescuing paths, can save lives.

As noted, ASARs can be reliable tools for disaster management, given their high technical capabilities and the ability to save time, costs, and manpower. Traditionally, ASARs were used as monitors for gathering needed data for helping in fast and accurate decision making of rescuing crews, but they also can be used in future to replace the role of humans in many risky tasks.

\section{CHALLENGES AND SOLUTIONS}

However, there are several challenges to overcome when designing successful ASAR. These challenges are as following:

1. Design of robust enough hardware that can resist collisions and is easy to transport,

2. Deployment of innovative sensors for safe navigation and rescuing purposes,

3. Reliability of robot with respect to accidental (safety) or malicious (security) risks,

4. Sufficient autonomy level both in terms of navigation and interpretation of the data sensed,

5. Designing new control methods for autonomous flight,

6. Fast interpretation and analysis of vital data collected through sensors. 
Solutions for the first challenge, that is, design of robust enough hardware may be the following [1]:

- Compact, small size and low weight electronics, without wired off-board sensors,

- Module-based design with slot-based architecture to achieve task specific application,

- Universal communication interface allowing the usage of both wireless (different RF modems) and wired (FTDI USB) connectivity,

- Autopilot which can store at least 200 waypoints, the parameters of planes, and control functions in a non-volatile memory,

- Fully autonomous operation (telemetry issues should not affect the flight).

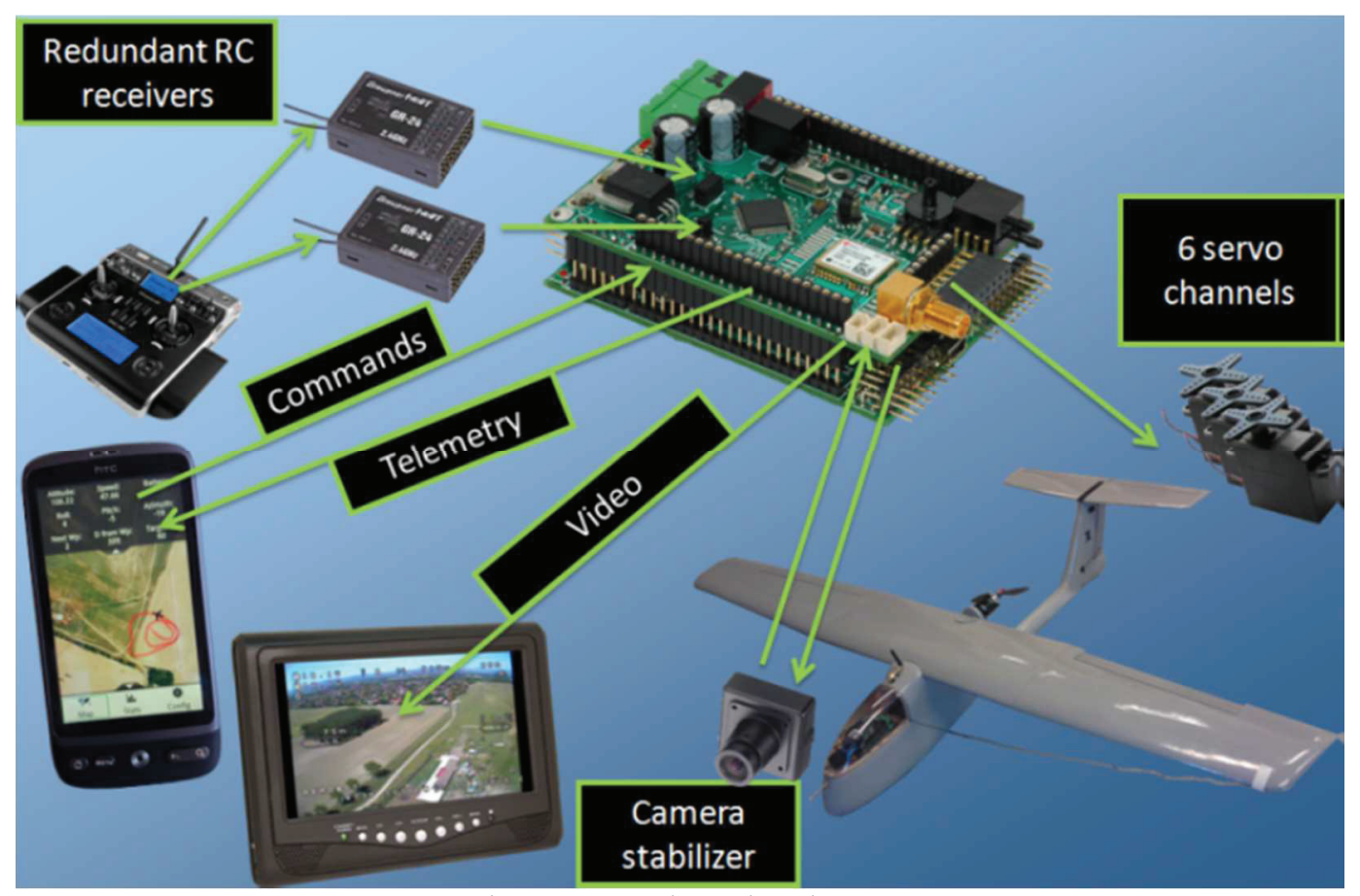

Figure 1. Robust hardware

Solutions for second challenge is development of new and innovative sensors, such as:

- Forward- and downward-facing vision sensors to detect obstacles,

- New types of cameras,

- Obstacle sensing,

- Air sensors for situational awareness,

- Collision avoidance sensors,

- Spotlights for dark or low-light areas,

- Loudspeakers. 


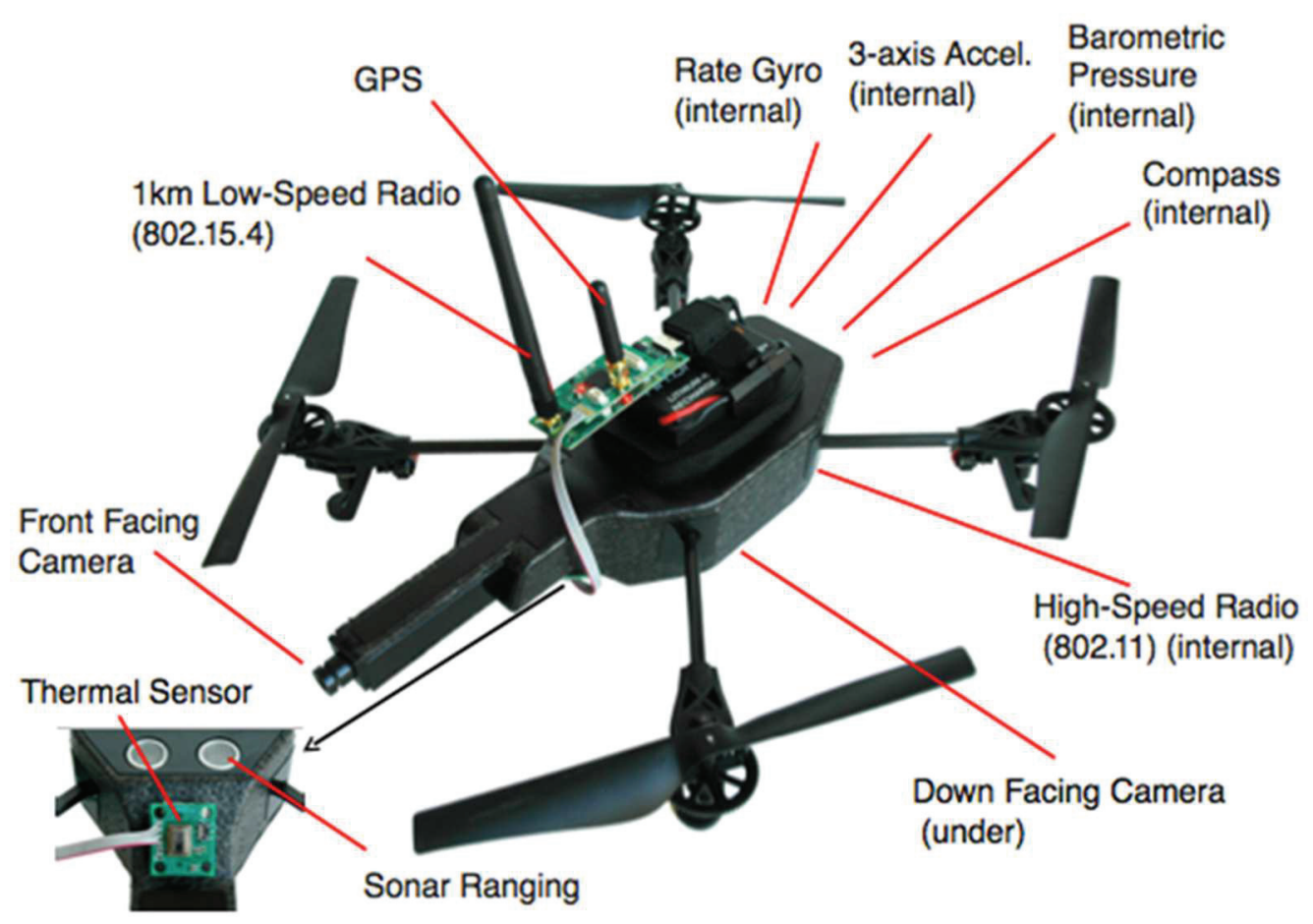

Figure 2. New and innovative sensors

Solutions for third challenge related to reliability of ASAR are as following:

- The reliability assessment must be performed on the ASAR for the main purpose of finding the baseline for the requirements of making the ASAR highly reliable,

- Each complex system such as an ASAR, being inserted in generally very extreme environment, must have its intrinsic reliability. Therefore, reliability evaluation of a system and its subsystems is an important task,

- The intrinsic reliability is the one that comes from a careful study of ASAR's parts so that there is a set of design criteria that take into account the vulnerabilities of the considered ASAR,

- Mean Time Between Failure (MTBF) and Failure Rate (FR) can suggest useful information about diagnostic aspects, maintenance and investments needed for design and production of a highly reliable ASAR,

- Obviously not all the parts or subsystems will have the same failure rate so not all parts or subsystems will be treated equally. It is possible to individuate the most critical components from the reliability point of view and thus suitably design them,

- The reliability model can be also used to assess key product parameters (voltage, temperature and so on) in order to perform devices stress and derating analyses. 




Figure 3. Failure rate (FR) model

To obtain solutions to fourth challenge autonomy classification model has to be applied. One of such models has four levels to classify autonomy of ASAR [2]:

- Level 1: Remotely Controlled System - System reactions and behavior depend on operator input,

- Level 2: Automated System - Reactions and behavior depend on fixed built-in functionality (preprogrammed),

- Level 3: Autonomous non-learning system - Behavior depends upon fixed built-in functionality or upon a fixed set of rules that dictate system behavior (goal-directed reaction and behavior),

- Level 4: Autonomous learning system with the ability to modify rules defining behaviors - Behavior depends upon a set of rules that can be modified for continuously improving goal directed reactions and behaviors within an overarching set of rules/behaviors.

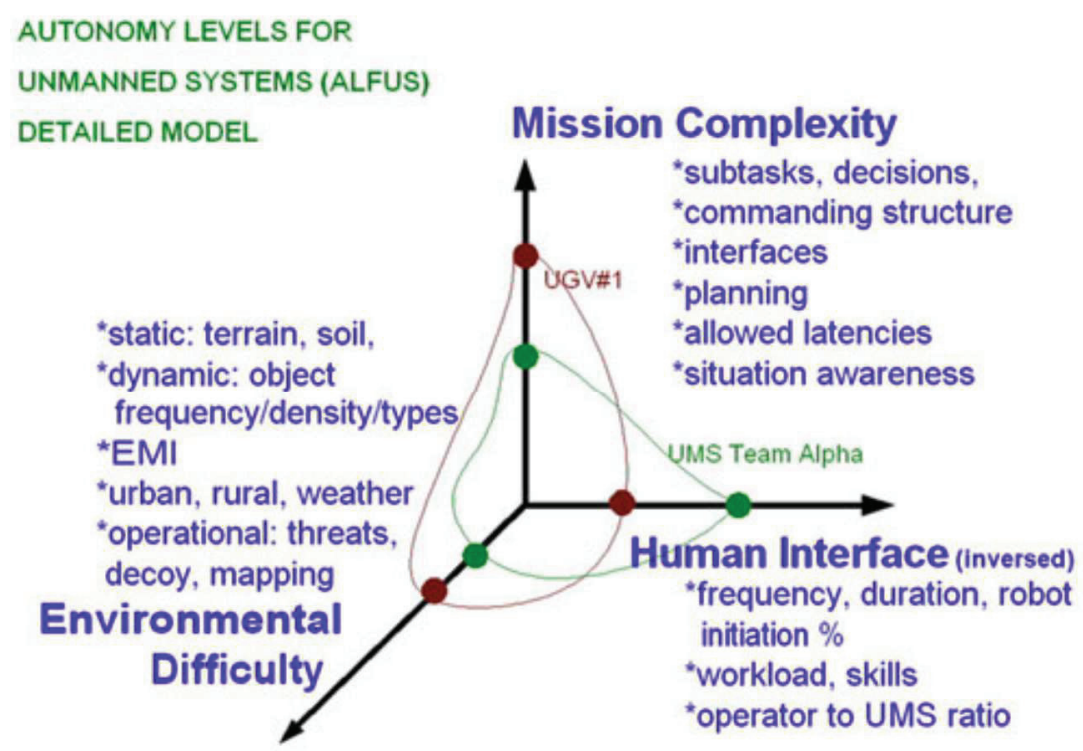

Figure 4. Autonomy levels - ALFUS model [2] 
New control methods as solution for fifth challenge may be applied as following [3]:

- ASARs are commonly used for missions in unknown environments, where an exact mathematical model of the environment may not be available,

- Using reinforcement learning (RL) to allow the ASAR to navigate successfully in such environments can bring improvements into control methods of ASAR. RL became popular recently thanks to its capabilities in solving learning problem without relying on a model of the environment,

- The ASAR could be controlled by altering the linear/angular speed, and the motion capture system provides the ASAR's relative position inside the space,

- Popular RL algorithm known as Q-learning, in which the agent computes optimal value function and records them into a tabular database, called Q-table, can be used. This knowledge can be recalled to decide which action it would take to optimize its rewards over the learning episodes. For each iteration, the estimation of the optimal state is made and value function is update,

- To carry out the algorithm, the ASAR should be able to transit from one state to another, and stay there before taking new action.

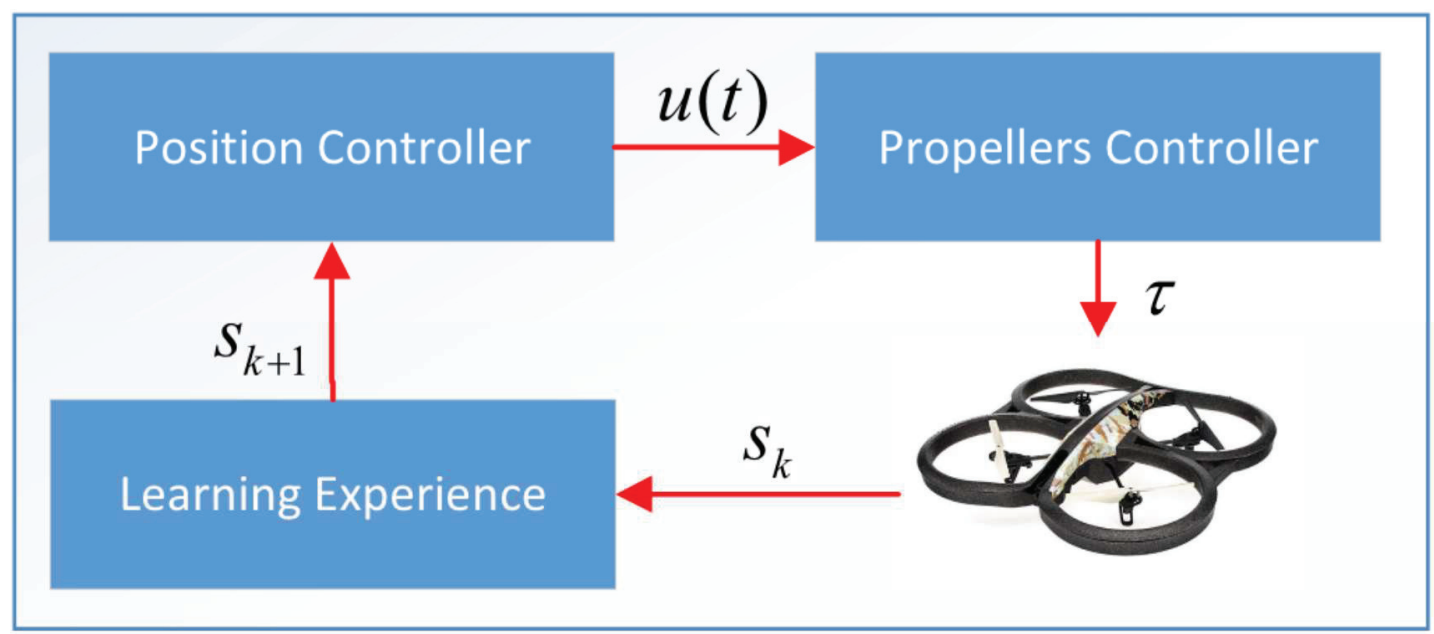

Figure 5. Reinforcement (RL) learning model [3]

Solutions for sixth challenge, fast interpretation and analysis of vital data collected through sensors, is Application of Big Data for Disaster Management. The storage and processing of large volumes of disaster data are perhaps the biggest challenges to be faced by civil defense, police, fire departments, public health and other government organizations managing disasters. It is very crucial for these organizations to get processed real-time disaster data as quickly as possible in order to react and coordinate efficiently all necessary activities in the cases of crises.

Big data tools and techniques can assist disaster management officials to optimize decisionmaking procedures. The tools and techniques that can be used may be:

- Data mining,

- Machine learning (ML),

- Real-time analytics,

- Cloud sourcing,

- Visualization. 




Figure 6. Big Data in rescuing missions

\section{EXAMPLE OF ASAR ROBOT}

InfoSet's ASAR robot Flying Tiger Model A1 was constructed having in mind requirements to solve some of challenges mentioned in previous text.

Flying Tiger Model A1 has a multi-rotor structure capable of delivering half-kilogram up to 5 kilograms of cargo depending on the mission defined for them. A summary of the characteristics of this ASAR is presented in Table 1 [4]:

\begin{tabular}{|c|c|}
\hline min 40 & Continuity of flight \\
\hline meter 500 & Operational flight altitude \\
\hline $\mathrm{km} 10$ & Operational radius \\
\hline $\mathrm{cm} 1000$ & Length Body Length \\
\hline $\mathrm{k} / \mathrm{g} 4 / 5$ & Robot's weight on take-off \\
\hline $\mathrm{g} 300$ & Maximum Optical Cargo Weight \\
\hline Electric 4 and 6 rotor & motors \\
\hline Up to 40 degrees and maximum wind of kph $25-10$ & environmental conditions \\
\hline
\end{tabular}

Table 1. Operating Characteristics of Systems

It also has following capabilities:

- It has possibility of fully automatic take-off,

- Its body has stable carbon structure,

- It is able to carry out day and night surveillance flights,

- It is able to perform aeronautical observations,

- It is easy to carry, 
- Its modular structure enables reaching very short operating conditions after deployment (less than 10 minutes),

- It has various flight modes for fully automated missions without the need for human agent involvement,

- For surveillance and identification, a digital camera and HD with a magnification of 10 times are used,

- In order to obtain stable images around the vertical axis of the flight path, a stabilizing system is used.

The land section, along with the airspace section, focuses on mission planning, flight control, and information processing. This part is responsible for sending and receiving information from the ASAR to rescuers and is actually user interface with the ASAR. The most important subsections included in this section are:

- Flight Planning,

- Flight Guidance and Control Station: includes Flight Design and Flight Management software, as well as Data Receiver,

- Monitoring station for receiving and displaying images from ASAR

The ground station, using the information provided, enables displaying of all the platform information, flight elements, and other parameters required by the users on the ground. It is also equipped with all necessary processing tools, such as keyboard, control arms and the like. The ground system is also equipped with USB ports for data entry or copying into the system.

The ground station software is equipped with an appropriate graphical interface for communication with the user, and along with the hardware capabilities of the platform, it is possible to design flight, change the direction of the ASAR and guide it during the mission. The flight control system provided on the platform along with the ground guidance software allows manual and autopilot flying or automatic guidance based on the use of GPS/INS. Also, to prevent control problems on the platform, it is possible to define the "home" for the ASAR and "return home" in crisis situations.

The ground station is designed as a native software installed on a laptop. In this way, the software used can be portable software that will be able to be carried and used as needed.

The second part of the station is receiving video images. In this section, images received from the ASAR are displayed based on the image receiver installed with the embedded antenna of ASAR. The images are displayed on the mounted monitor.
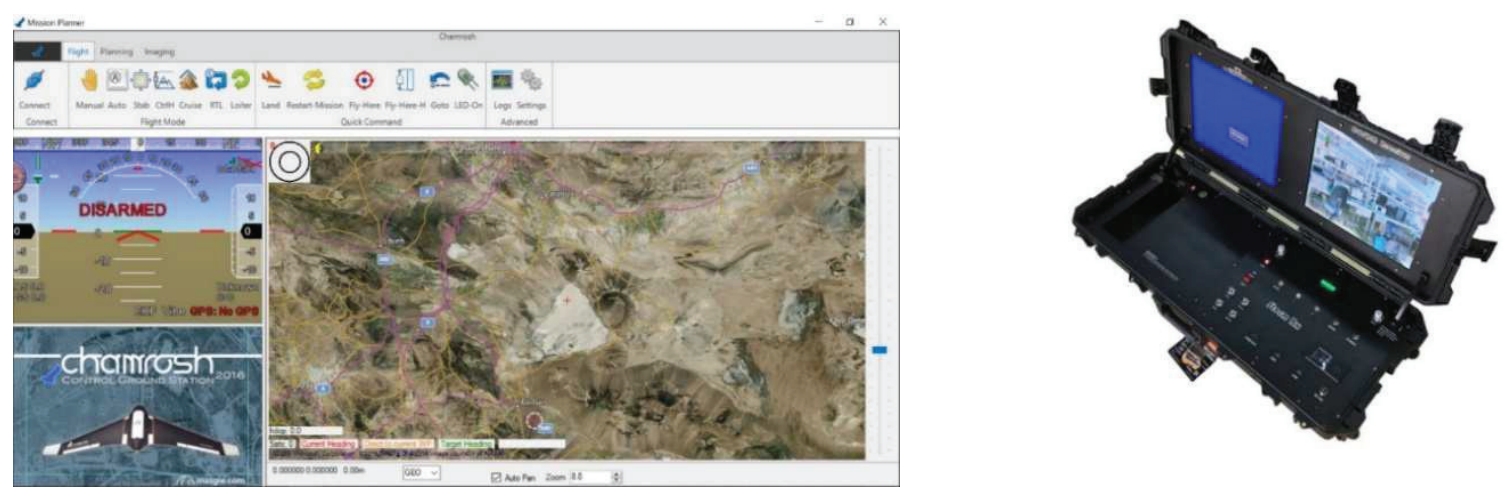

Figure 7. The ground station 


\section{CONCLUSIONS}

For six known challenges related to improving ASAR's efficiency in disaster situations, we presented current efforts in finding relevant solutions.

Some solutions are easier to be found and implemented, while others might require more research and development, especially in domains of developing new control methods and obtaining fast interpretation and analysis of vital data collected through sensors.

New methods, such as reinforcement learning (RL), data mining, machine learning (ML), realtime analytics, cloud sourcing, visualization, and other Big Data tools and techniques can assist disaster management to optimize control efficiency of ASAR and decision-making procedures for ground personnel.

We also presented some of characteristics of InfoSet's ASAR robot Flying Tiger Model A1 which was constructed considering requirements for solving some of challenges mentioned in the article. Further work and development on this type of ASAR will be performed by InfoSet's robotic team in near future.

\section{REFERENCES}

[1] Dániel Stojcsics, András Molnár, (2012) Air Guardian - UAV Hardware and Software System for Small Size UAVs, Regular Paper, Open Access Article

[2] Marco Protti, (2007) UAV Autonomy - Which Level is Desirable? - Which Level is Acceptable? Alenia Aeronautica Viewpoint, NATO/OTAN Unclassified/Unlimited document

[3] Huy X. Pham, Hung. M. La, David Feil-Seifer, Luan V. Nguyen, (2018) Autonomous UAV Navigation Using Reinforcement Learning, arXiv:1801.05086v1 [cs.RO]

[4] InfoSet Flying Tiger Model A1 specifications (2018), InfoSet 